\title{
Development of a semi-defined medium supporting production of platensimycin and platencin by Streptomyces platensis
}

\author{
Sabrina Aluotto, Heather Tynan, Christian Maggio, Maria Falzone, Anisha Mukherjee, Vincent Gullo \\ and Arnold L Demain
}

Platensimycin and platencin are compounds that were discovered at Merck Research Laboratories and have shown promising antibacterial activity. They are both produced in fermentation by the actinomycete Streptomyces platensis. Merck reported a crude, insoluble production medium to produce the antibiotics. To test the possible effects of different primary metabolites and inorganic compounds on the production of these antibiotics, a chemically-defined medium is needed. The effects that these compounds have on production could provide information about the precursors and biosynthetic pathway of the antibiotics. We have tested and developed a number of media with varying degrees of chemical definition and solubility using the Merck medium as our starting point. Our latest production medium, PM5, is soluble and semi-defined. It yields suitable production of the compounds, as shown by agar diffusion assays, bioautography and HPLC. The antibiotics were located in the extracellular broths and not in the mycelia.

The Journal of Antibiotics (2013) 66, 51-54; doi:10.1038/ja.2012.97; published online 28 November 2012

Keywords: antibiotics; media; platencin; platensimycin

\section{INTRODUCTION}

Two potentially important antibiotics are platensimycin and platencin. ${ }^{1}$ They were discovered at Merck Research Laboratories ${ }^{2}$ and showed potent antibacterial activity as well as low toxicity, implying great potential. Platensimycin was isolated from a soil sample originating from South Africa in $2006 .{ }^{3-5}$ It was found to be produced by the actinomycete Streptomyces platensis strain MA7327.1,2 Merck scientists tested the compound and found it to interfere with fatty acid synthesis by inhibiting the FabF enzyme of the type II system. ${ }^{6}$ The molecule is hydrophobic and contains 3-amino-2,4-dihydroxybenzoic acid and a C-17 pentacyclic enone with an ether ring. ${ }^{2}$ These two moieties are joined by an amide bond. Platencin, a structurally related compound, was isolated from a soil sample originating from Spain and was found to be produced by S. platensis strain MA7339. It also consists of an aminobenzoic acid moiety connected to a tetracyclic unit by an amide bond; however, in platencin, the tetracyclic unit does not contain an ether ring. ${ }^{7}$

Platencin kills bacteria by inhibiting fatty acid biosynthesis via inhibition of the enzymes FabF and FabH of the type II system. ${ }^{5}$ Both compounds, platencin and platensimycin, are active against Gram-positive bacteria and resistant strains such as vancomycinresistant enterococci and both linezolid- and macrolide-resistant pathogens. $^{5}$ Platensimycin also is active against Mycobacterium tuberculosis. ${ }^{8}$
Prokaryotes and eukaryotes carry out different types of fatty acid synthesis. Prokaryotes use the type II fatty acid synthesis system, whereas eukaryotes, for example, humans, carry out type I fatty acid synthesis. This difference is extremely important for the potential of these antibiotics because they specifically target type II fatty acid synthesis and thus would not be expected to be toxic to humans. ${ }^{9}$

The antibiotics were produced in fermentation at Merck using an insoluble, complex medium, which contains Amberex, yellow cornmeal and lactose. ${ }^{1}$ Our goal is to investigate the effects of primary metabolites and inorganic compounds on the synthesis of the antibiotics. The best way to observe this would be in a chemicallydefined production medium, which has not been reported in the literature. The present publication discloses our successful efforts in devising a soluble, semi-defined production medium that supports antibiotic production. Future studies will focus on further development of this medium into a chemically-defined production medium.

\section{MATERIALS AND METHODS}

Stock, seed and fermentation cultures

S. platensis strain MA7327, which produces both platensimycin and platencin, was obtained from Merck \& Co., Inc (Rahway, NJ, USA). It was grown in a stock culture medium, which contains $\left(\mathrm{gl}^{-1}\right) 30$ Difco Trypticase Soy Broth (Difco Laboratories, Detroit, MI, USA) solids in deionized water. The medium 
was distributed into $250 \mathrm{ml}$ flasks at $50 \mathrm{ml}$ per flask, autoclaved and inoculated with a sample of $S$. platensis MA7327. The inoculated Trypticase Soy Broth flask was placed on a gyratory shaker at 220 r.p.m. and $36^{\circ} \mathrm{C}$ for 5 days and used to inoculate a seed medium. The Merck seed medium consists of $\left(\mathrm{gl}^{-1}\right)$ 20 soluble starch, 10 dextrose, $5 \mathrm{NZ}$ amine type A, 3 Difco beef extract, 5 Difco Bacto Peptone, 5 Difco yeast extract and $1 \mathrm{CaCO}_{3}$. Before the addition of the $\mathrm{CaCO}_{3}$, the $\mathrm{pH}$ was adjusted to 7 . The seed flasks were inoculated with a wire loopful of the S. platensis MA7327 stock culture and were incubated on the gyratory shaker at 220 r.p.m. and $28^{\circ} \mathrm{C}$ for a period of 5 days.

The original insoluble, complex fermentation medium developed by Merck (a production medium, which we named PM1) consists of $\left(\mathrm{gl}^{-1}\right) 5$ Amberex, 40 yellow cornmeal and 40 lactose. $^{1}$ All experiments were conducted in $250 \mathrm{ml}$ Erlenmeyer flasks containing $50 \mathrm{ml}$ of medium. The $\mathrm{pH}$ of all production media used in this work was adjusted to 7.0. The flasks were inoculated with $5 \mathrm{ml} \mathrm{S}$. platensis seed culture and incubated on a gyratory shaker at 220 r.p.m. and $28^{\circ} \mathrm{C}$.

\section{Zone of inhibition assay}

In order to test for antibiotic productivity, agar plates inoculated with Staphylococcus aureus (85W1178 from Ward's Natural Science) were used. The plates were made by combining $3.7 \mathrm{~g}$ of EMD LB Agar MILLER solids with $100 \mathrm{ml}$ of deionized water. The mixture was autoclaved and allowed to cool before inoculating with $2.5 \mathrm{ml}$ of a stock culture of $S$. aureus. The inoculated mixture was placed into Petri dishes at $10 \mathrm{ml}$ per dish. To test for antibiotic production, paper disks were dipped into the S. platensis MA7327 fermentation broths, laid out to dry on aluminum foil, placed on the S. aureus agar plate and incubated overnight at $35^{\circ} \mathrm{C}$. Two disks per fermentation flask were used; all fermentations were run in duplicate. The disk assay was carried out for a number of days throughout the course of fermentation. The data given are averages of the duplicate flask results.

\section{Chemical (quantitative HPLC) assay}

For quantitative analysis, a $25 \mathrm{ml}$ portion of 6-day fermentation broths was centrifuged in each of four $50 \mathrm{ml}$ centrifuge tubes. The supernatants were combined and the $\mathrm{pH}$ was adjusted to $\sim 5$ with $1.2 \mathrm{M}$ hydrochloric acid. It was then extracted three times with an amount of ethyl acetate equal to the volume of supernatant. Both the extract and spent aqueous solution were tested for antibacterial activity to ensure that all the antibiotics had been extracted. To test the mycelial layer of the centrifuged fermentation broth for antibiotics, $15 \mathrm{ml}$ of acetone were added to the cells and the mixture was transferred to a round bottom flask and stirred for $30 \mathrm{~min}$. It was then centrifuged to remove the cells and the acetone was evaporated. The remaining aqueous solution was then extracted with an equal volume of ethyl acetate. The extract and spent solution were tested for antibacterial activity.

TLC was used to detect the presence of platensimycin and platencin in the samples. Control samples of platensimycin and platencin were spotted along with the ethyl acetate extract from the mycelia and from the supernatant. The plate was developed with $90 \%$ dichloromethane and $10 \%$ methanol. The TLC plate was then tested for antibacterial activity using bioautography to elute the compounds into agar plates inoculated with $S$. aureus. The plates were incubated overnight (as described above) and observed for zones of inhibition.

HPLC of a platensimycin and platencin control dissolved in methanol and the ethyl acetate extracts of the supernatant and mycelia of the fermentation were chromatographed on a $\mathrm{C}_{18}$ column (Phenomenex 5 micron $\mathrm{C}_{18}$, $150 \times 4.6 \mathrm{~mm}$ ) and eluted with a linear gradient from $15 \%$ acetonitrile/ aqueous $0.01 \%$ trifluoroacetic acid to $95 \%$ acetonitrile/aqueous $0.01 \%$ trifluoroacetic acid. All samples were run for $20 \mathrm{~min}$ at a flow of $1 \mathrm{ml} \mathrm{min}^{-1}$. Detection of compounds was measured at $254 \mathrm{~nm}$. The quantity of platensimycin and platencin in the fermentation extracts was determined by comparing the concentrations to the reference standard using peak heights.

\section{RESULTS}

Production in insoluble complex medium

Before testing any new media, we grew $S$. platensis in the original insoluble and complex Merck production medium (PM1) to ensure that we would observe growth and antibiotic production. The results in Figure 1 confirm the ability of PM1 to support antibiotic production.

Production in a soluble complex media

Our first goal was to devise a soluble, although still complex, medium. We tested two such media (production medium 2: PM2 and production medium 3: PM3). In $\mathrm{gl}^{-1}$, PM2 was composed of 26.5 Difco Tyrpticase Soy Broth without dextrose, 10 soluble starch, and 20 MOPS buffer in deionized water. PM3 consisted of $\left(\mathrm{gl}^{-1}\right) 40$ dextrin, 40 lactose, 5 yeast extract and 20 MOPS buffer in deionized water. The results of an experiment comparing PM1, PM2 and PM3 are shown in Table 1. Both soluble complex media supported growth and antibiotic production. PM3 appeared slightly better than PM2.

\section{Production in soluble semi-defined media}

Our initial semi-defined production medium (PM4) contained two different types of chemically-defined supplements from Difco Laboratories, which were added to the dextrin, MOPS buffer and lactose components of PM3. The supplements were used as a means to replace the yeast extract of medium PM3. The first supplement was Yeast Carbon Base, which contains dextrose, three amino acids, nine vitamins, seven trace elements and four inorganic salts. The second was Vitamin-Free Yeast Base, which contains dextrose, three amino acids, seven trace elements, four inorganic salts and one nitrogen source (ammonium sulfate). Thus, production medium 4 (PM4), contained $\left(\mathrm{g} \mathrm{l}^{-1}\right) 40$ dextrin, 40 lactose, 5 Difco Yeast Carbon Base, 5 Difco Vitamin-Free Yeast Base and 20 MOPS buffer in deionized water. Production in PM4 and PM3 was compared with that of the Merck production medium (PM1). Figure 2 shows that semi-defined PM4 gave excellent results.

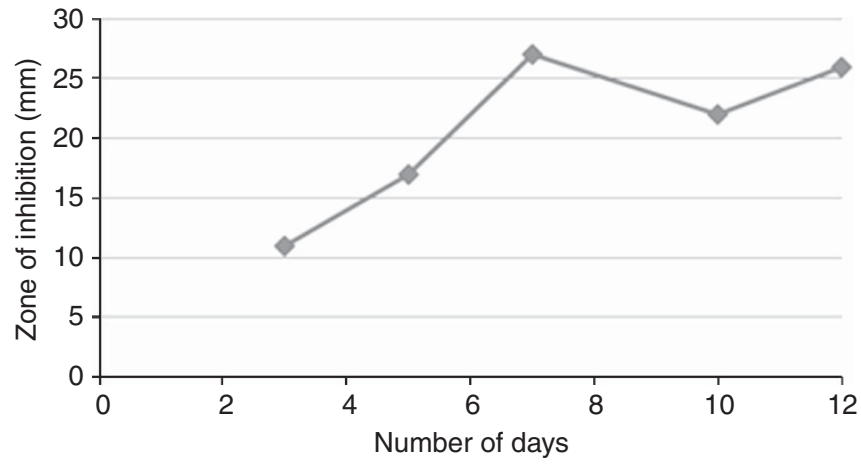

Figure 1 Zones of inhibition against $S$. aureus produced by $S$. platensis grown in Merck insoluble complex production medium PM1. A full color version of this figure is available at The Journal of Antibiotics journal online.

Table 1 Comparison of the inhibitory zones observed against $S$. aureus in Merck complex, insoluble medium PM1 and soluble complex media PM2 and PM3

\begin{tabular}{lccc}
\hline & \multicolumn{3}{c}{ Zone size $(\mathrm{mm})$} \\
\cline { 2 - 4 } Medium & Day 3 & Day 7 & Day 8 \\
\hline PM1 & 28 & 28 & 30 \\
PM2 & 25 & 23 & 24 \\
PM3 & 27 & 28 & 25 \\
\hline
\end{tabular}

Abbreviation: PM, production medium 


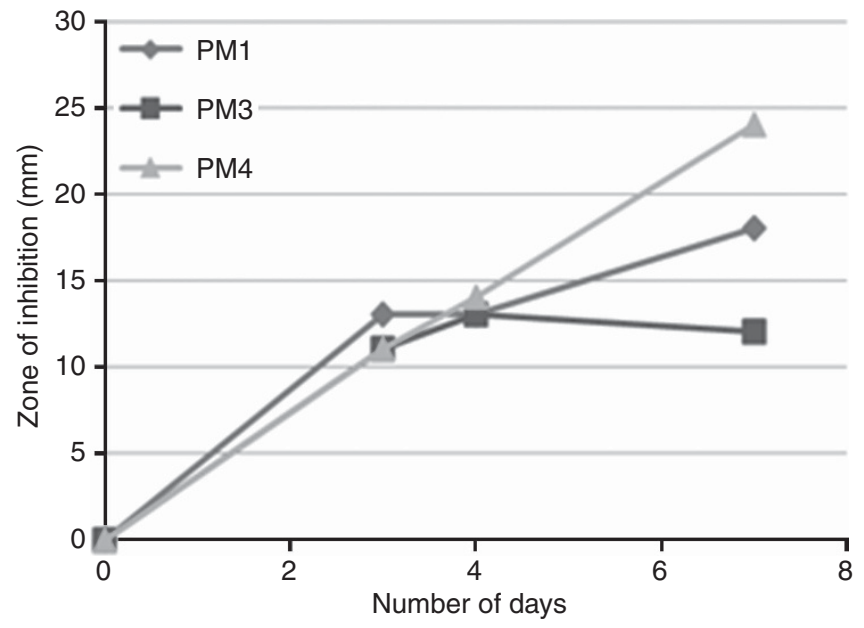

Figure 2 Zones of inhibition against $S$. aureus observed during fermentation in Merck complex insoluble medium PM1, soluble complex medium PM3 and soluble semi-defined medium PM4. A full color version of this figure is available at The Journal of Antibiotics journal online.

Table 2 Comparison of inhibition zones during fermentation in media PM4, PM4A and PM4B

\begin{tabular}{lccccccc}
\hline & \multicolumn{7}{c}{ Zone size $(\mathrm{mm})$} \\
\cline { 2 - 7 } Medium & Day 0 & Day 1 & Day 4 & Day 5 & Day 7 & Day 8 & Day 12 \\
\hline PM4 & NZ & NZ & 10 & 13 & 22 & 16 & 23 \\
PM4A & NZ & NZ & 11 & 24 & 24 & 19 & 26 \\
PM4B & NZ & NZ & NZ & NZ & NZ & NZ & NZ \\
\hline
\end{tabular}

Abbreviations: PM, production medium; NZ, no zone.

Table 4 Composition of media used in this study

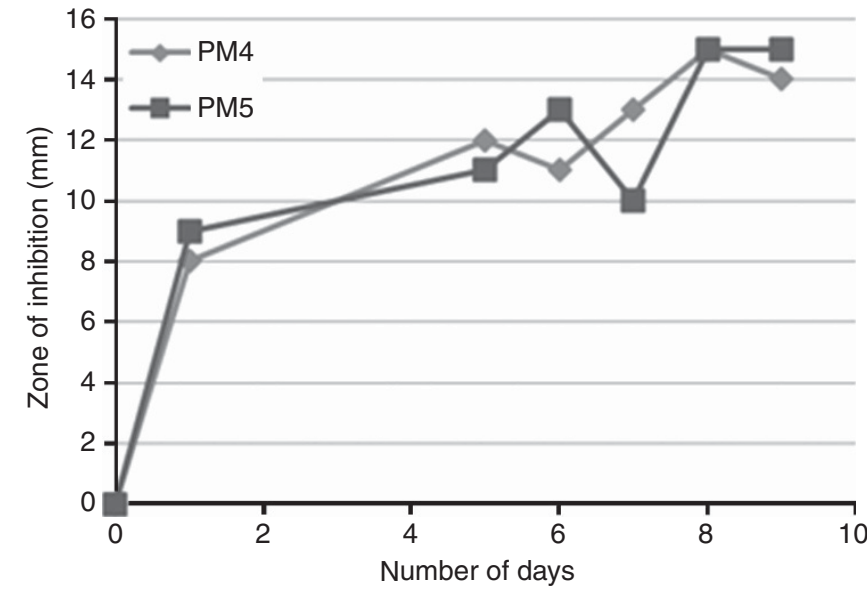

Figure 3 Zones of inhibition against $S$. aureus observed during fermentation in semi-defined media PM4 and PM5. A full color version of this figure is available at The Journal of Antibiotics journal online.

Table 3 HPLC assays of platensimycin and platencin produced in media PM1, PM4 and PM5

\begin{tabular}{lcc}
\hline Medium & Platensimycin & Platencin \\
\hline PM1 & $2.22 \mu \mathrm{g} \mathrm{ml}^{-1}$ & $0.77 \mu \mathrm{g} \mathrm{ml}^{-1}$ \\
PM4 & $8.28 \mu \mathrm{gl}^{-1}$ & $2.92 \mu \mathrm{gl}^{-1}$ \\
PM5 & $5.13 \mu \mathrm{g} \mathrm{ml}^{-1}$ & $1.81 \mu \mathrm{g} \mathrm{ml}^{-1}$ \\
\hline
\end{tabular}

Abbreviation: PM, production medium.

\begin{tabular}{|c|c|c|c|c|c|c|c|}
\hline Component & \multicolumn{7}{|c|}{ Medium } \\
\hline Yellow corn meal & 40 & - & - & - & - & - & - \\
\hline Lactose & 40 & & 40 & 40 & 40 & 40 & 40 \\
\hline Difco Trypticase Soy Broth without dextrose & - & 26.5 & - & - & - & - & - \\
\hline Dextrin & - & - & 40 & 40 & 40 & 40 & 40 \\
\hline Yeast extract & - & - & 5 & - & - & - & - \\
\hline Difco Yeast Carbon Base & - & - & - & 5 & - & 5 & - \\
\hline Difco Vitamin-Free Yeast Base & - & - & - & 5 & 5 & - & - \\
\hline Ammonium sulfate & - & - & - & - & - & 2 & 2 \\
\hline
\end{tabular}

With the success of medium PM4, our next goal was to develop a simpler soluble, semi-defined medium because medium PM4 contains 28 components. It was simplified by adding only one of the two Difco supplements resulting in media PM4A and PM4B. PM4A contained $\left(\mathrm{gl}^{-1}\right) 40$ dextrin, 40 lactose, 5 Difco Vitamin-Free Yeast Base and 20 MOPS buffer in deionized water. PM4B contained $\left(\mathrm{gl}^{-1}\right)$ 40 dextrin, 40 lactose, 5 Difco Yeast Carbon Base and 20 MOPS buffer, in deionized water. The next experiment compared PM4,
PM4A and PM4B for production of antibiotics. The results are shown in Table 2. Semi-defined medium 4A supported very potent production, more so than medium 4 , whereas semi-defined medium $4 \mathrm{~B}$ did not support production.

To attempt medium simplification, the Difco Vitamin-Free Yeast Base in medium $4 \mathrm{~A}$ was replaced by ammonium sulfate. This very simple production medium 5 (PM5) contained $\left(\mathrm{gl}^{-1}\right) 40$ dextrin, 40 lactose, 2 ammonium sulfate and 20 MOPS buffer, in deionized water. 
In a series of experiments, PM5 was found to be a successful soluble, semi-defined production medium. An example is shown in Figure 3.

To confirm that the antibacterial activity observed with our semidefined media was due to platensimycin and platencin produced in fermentation, bioautography and HPLC assays were run on 7-day samples. PM1, PM4 and PM5 were analyzed. Bioautography clearly showed that platensimycin and platencin were produced in all three fermentation media. The results from quantitative HPLC analysis are shown in Table 3. As expected, platensimycin and platencin were produced and the ratio was $3: 1$, respectively, in all media. Platencin and platensimycin were undetectable in the mycelial extract.

\section{DISCUSSION}

We were successful in achieving our immediate goal, which was to obtain a simple, semi-defined medium for production of platensimycin and platencin. These two antibiotics were found in the extracellular broth but not in the mycelia. A future step will be development of a chemically-defined medium to which we can add inorganic compounds and various primary metabolites, such as amino acids, vitamins, organic acids and nucleosides, and observe any positive or negative effects they have on the production of the antibiotics. This could provide us with information about the biosynthetic pathway, its precursors and its regulation.

Most of the media devised and used here supported antibiotic production. The composition of all the media is shown in Table 4.We were successful in introducing lactose, dextrin and MOPS buffer in PM2 and PM3; all except dextrin are soluble and chemically-defined, whereas dextrin is soluble and semi-defined. In PM4, PM4A and PM4B, we introduced two Difco products to provide nutrients in a chemically-defined and readily available manner. Replacing both Difco products with ammonium sulfate was surprisingly successful and greatly simplified the medium. Although PM5 did not yield as much antibiotic as PM4 as determined by HPLC, PM5 is a simple, soluble medium containing only four components as compared with PM4 with 28 components.
In this study, we have created a simple medium that is soluble, semi-defined and able to support antibiotic production (PM5). Although not yet chemically-defined, we are now in a good position to create a simple, soluble, chemically-defined medium for platensimycin and platencin production.

\section{ACKNOWLEDGEMENTS}

We thank Dr Sheo Singh of Merck \& Co., Inc. and Dr Ben Shen of Scripps, Florida for their gifts of $S$. platensis cultures. We also thank Dr Shen for the samples of platensimycin and platencin. The $S$. aureus test culture was a gift from Dr Joanna Miller of Drew University. This work was supported by the Drew Summer Science Institute (DSSI) and RISE. We acknowledge the encouragement by former RISE Director Dr Ashley H Carter (now deceased), the present Director Dr Jon Kettenring and RISE administrator Miriam Donohue. Authors SA, HT, CM, and MF were Drew undergraduate students. AM was a high school student.

1 Singh, S. et al. Isolation, structure, and absolute stereo-chemistry of platensimycin, a broad spectrum antibiotic discovered using an antisense differential screening strategy. J. Am. Chem. Soc 128, 11916-11920 (2006).

2 Martens, E. \& Demain, A. L. Platensimycin and platencin: promising antibiotics for future application in human medicine. J. Antibiot. 64, 705-710 (2011).

3 Young, K. et al. Discovery of FabH/FabF inhibitors from natural products. Antimicrob. Ag. Chemother. 50, 519-526 (2006).

4 Wang, J. et al. Platensimycin is a selective FabF inhibitor with potent antibiotic properties. Nature 441, 358-361 (2006).

5 Wang, J. et al. Discovery of platencin, a dual FabF and FabH inhibitor with in vivo antibiotic properties. Proc. Natl Acad. Sci. USA 104, 7612-7616 (2007).

6 Singh, S. B., Phillips, J. W. \& Wang, J. Highly sensitive target-based whole-cell antibacterial discovery strategy by antisense RNA silencing. Curr. Opin. Drug Discov. Devel. 10, 160-166 (2007).

7 Jayasuriya, H. et al. Isolation and structure of platencin: FabH and FabF dual inhibitor with potent broad-spectrum antibiotic activity.. Angew. Chem. Int. Ed. Engl. 46, 4684-4688 (2007).

8 Potera, C. Novel pentacyclic from soils blocks bacterial fatty acid synthesis. Microbe 1 , 350-351 (2006).

9 Payne, D. J., Warren, P. V., Holmes, D. J. \& Yi, Y. Bacterial fatty-acid biosynthesis: genomics-driven target for antibacterial drug discovery.. Drug Disc. Today 6, 537-544 (2001). 